\title{
El trabajo interdisciplinario de los equipos base del proyecto pedagógico para las personas menores de edad en albergues del Patronato Nacional de la Infancia
}

\author{
The Interdisciplinary Work Among the Base Teams of Professionals in a Pedagogical Project for \\ Institutionalized Children in the Children National Protection System (Patronato Nacional De La Infancia) \\ O trabalho interdisciplinar das equipes de base do Projeto Pedagógico para pessoas menores de \\ idade em abrigos do Patronato Nacional de la Infancia
}

Roxana Rodríguez-Araya Universidad Nacional

ROR:: https://ror.org/01t466c14 Heredia, Costa Rica roxana.rodriguez@una.cr

http://orcid.org/0000-0001-6634-9780

Priscilla Carranza-Marchena Universidad Nacional

ROR:..https://ror.org/01t466c14 Heredia, Costa Rica priscilla.carranza.marchena@una.ac.cr https://orcid.org/0000-0002-0742-7328

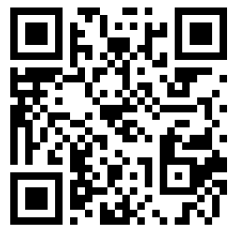

Raquel Bulgarelli-Bolaños Universidad Nacional

ROR:: https://ror.org/01t466c14 Heredia, Costa Rica raquelbulgarelli@gmail.com http://orcid.org/0000-0002-8811-9625

Silvia Ulate-Oviedo

Universidad Nacional

ROR:: https://ror.org/01t466c14

Heredia, Costa Rica silviaulate@gmail.com

(iD) https://orcid.org/0000-0001-5369-9597

Maureen Camacho-Oviedo

Universidad Nacional

ROR:: https://ror.org/01t466c14

Heredia, Costa Rica

maureen.camacho.oviedo@una.cr

https://orcid.org/0000-0002-1827-0928

Recibido • Received • Recebido: 21 / 11 / 2018

Corregido • Revised • Revisado: 30 / 03 / 2020

Aceptado • Accepted • Aprovado: 07 / 07 / 2020 
http://doi.org/10.15359/ree.24-3.8

http://www.una.ac.cr/educare

educare@una.cr

Resumen: El proyecto pedagógico para las personas menores de edad ubicadas en albergues institucionales del PANI, concibe, como parte de su estrategia, acciones que orientan y promuevan el trabajo interdisciplinario en los equipos base de profesionales que trabajan en los cuarenta albergues que atiende el proyecto a nivel país. Este proyecto busca el fortalecimiento de habilidades académicas y emocionales de la población infantil y adolescente albergada, por medio del apoyo educativo de docentes en nueve disciplinas educativas que trabajan con ellos y ellas. Por tanto, el objetivo de este documento es evaluar el trabajo interdisciplinario del proyecto, desde la mirada profesional, en procura de comprender los esfuerzos que se han realizado en la praxis, de forma interdisciplinaria, en pro del fortalecimiento de las habilidades de la población atendida. Por tanto, el enfoque del estudio es cualitativo, de tipo descriptivo. En el análisis de la información se consideran las minutas de reunión de los grupos profesionales; además, encuestas con preguntas abiertas realizadas a los profesionales y las profesionales desde el 2016-2018. Como resultado, se rescata que la estrategia ha sido valorada mayoritariamente de manera positiva por los mismos cuerpos docentes y ha permitido crear sinergias interesantes por el bien de la población apoyada.

Palabras claves: Interdisciplinario; pedagogía; proyecto; profesionales; personas menores de edad.

Abstract: The pedagogical project for children and teenagers under the age of 18 years, who are institutionally sheltered at the Children National Protection System (PANI), conceives, as part of its strategies, all the actions guiding and promoting the interdisciplinary work of the teachers who work at the 40 shelter-homes the project works for along Costa Rica. This project seeks to strengthen the academic and emotional skills of these children and teenage population through the teachers' support, who work with them, in nine different academic disciplines. Thus, the objective of this study is to evaluate the interdisciplinary work from the perspective of the professionals; it tries to understand the efforts done in the praxis to strengthen children and adolescents' skills. The study used a qualitative and descriptive design. The analysis of the information considered the meeting minutes and open-ended questions from a questionnaire administered from 2016 to 2018. As a result, it is relevant to state that teachers positively valued this experience, and it has allowed interesting synergies for the children's common good.

Keywords: Interdisciplinary; pedagogy; project; professionals; minors.

Resumo: O projeto educativo para menores de idade em abrigos institucionais do PANI, tem como parte de sua estratégia ações para orientar e promover o trabalho interdisciplinar nas equipes profissionais que trabalham nos quarenta abrigos que o projeto atende no país. Esse projeto procura fortalecer as capacidades acadêmicas e emocionais da população menor de idade, através do apoio educacional de professores em nove disciplinas. Portanto, o objetivo deste documentoéavaliar otrabalho interdisciplinar do projeto sob a ótica dos profissionais, procurando entender de maneira interdisciplinar os esforços empreendidos na práxis, a fim de fortalecer as habilidades da população atendida. Portanto, o foco do estudo é qualitativo, descritivo. $\mathrm{Na}$ análise das informações, são consideradas as atas das reuniões dos profissionais; também pesquisas com perguntas abertas feitas aos profissionais a partir de 2016 a 2018. Como resultado, a estratégia tem sido valorizada de forma positiva pelos próprios professores e tem permitido criar sinergias interessantes para o benefício da população apoiada.

Palavras-chave: Interdisciplinaridade; pedagogia; projeto; profissionais; pessoas menores de idade. 


\section{Introducción}

Desde el año 2015, cuatro unidades académicas de la Universidad Nacional en Costa Rica, de ellas tres del Centro de Investigación y Docencia en Educación (CIDE) y una de la Escuela de Movimiento Humano y Calidad de Vida, se unieron para realizar un proyecto ambicioso, que implicaba la coordinación de esfuerzos en pro de la mejora de la experiencia educativa de la población infantil y adolescente que reside en los albergues del Patronato Nacional de la Infancia (PANI), centros concebidos como instituciones, entidades o espacios físicos donde se concentra el resguardo de personas menores de edad en condiciones vulnerables, cuya tutela temporal y salvaguarda es ejercida de manera transitoria o permanente por el Estado costarricense. De ahí que, este proyecto surge como respuesta a la inquietud del PANI por proveer una atención integral a los niños, niñas y adolescentes institucionalizados. Asimismo, la experiencia interdisciplinaria ha demandado la conjunción de profesionales de las unidades académicas participantes, para construir y dar seguimiento a la propuesta pedagógica planteada por el proyecto. Este trabajo conjunto ha favorecido la construcción de estrategias desde las diferentes disciplinas.

Adicionalmente, cabe indicar que este proyecto requirió de diferentes niveles de interdisciplinariedad. Uno de ellos es el objeto de este artículo, el cual se concentra en evaluar el trabajo interdisciplinario del proyecto desde la perspectiva de los profesionales y las profesionales, procurando comprender los esfuerzos que se han realizado en la praxis de forma interdisciplinaria en pro del fortalecimiento de las habilidades de la población atendida. Al inicio se le planteó al equipo docente una propuesta pedagógica base, pero interpretada por los docentes y las docentes, quienes la reorganizan y contextualizan según cada una de las regiones y albergues; por ende, el trabajo que se desarrolla es, en esencia, flexible.

Por otra parte, el proyecto promueve y solicita a los equipos de profesionales que se trabaje de forma interdisciplinaria en la mayor medida posible, con el fin de que las acciones realizadas permitan un alcance más amplio dentro del marco de la colectividad. Por todo esto, el presente artículo profundiza en mostrar la propuesta pedagógica y su interpretación desde el marco de la interdisciplinaridad, desarrollada por el grupo de profesionales de los equipos base de los albergues, además de mostrar el impacto de estos esfuerzos en la mejora de la experiencia educativa de la población atendida.

\section{Marco referencial}

\section{Contextualizando la atención educativa en el marco del proyecto pedagógico}

El equipo de profesionales que trabajan en cada una de las alternativas de cuido o albergues pertenecen a diferentes disciplinas; a saber: orientación, pedagogía en I y II ciclos, educación especial, educación preescolar, educación física, educación musical, educación artística, enseñanza del inglés y enseñanza de la matemática. Estos profesionales asisten a los 
http://doi.org/10.15359/ree.24-3.8

http://www.una.ac.cr/educare

educare@una.cr

albergues en horas parciales, con el fin de apoyar los procesos formales o no formales en los que se encuentran las personas menores de edad (PME); por ejemplo, la docente de I y II ciclos asiste ocho horas semanales al albergue para apoyar la realización de tareas, preparación para pruebas escritas, además de atender necesidades como el desarrollo de la lectoescritura o la comprensión matemática; este abordaje es adicional a la participación académica de las PME en la escuela. Este acompañamiento es similar para el resto de profesionales de materias académicas, como en enseñanza del inglés, matemática, educación preescolar o educación especial; adicionalmente, docentes en el área de orientación, arte, educación física y música potencian el aprendizaje de habilidades más enfocadas al desarrollo del talento y disfrute personal.

El equipo de profesionales que trabajan en los albergues son personas especializadas en educación que, por lo general, residen en las comunidades en las que se encuentran las alternativas de protección; el trabajo que realizan debe estar de acuerdo con las características de la población. Entre esas características, se encuentran personas menores de edad migrantes, madres adolescentes, hombres y mujeres adolescentes y población infantil, con compromisos cognitivos, físicos o emocionales. La población menor de edad atendida por el proyecto se ubica en las siete provincias del país, ubicadas en 40 albergues a nivel nacional.

\section{La propuesta pedagógica desde la interdisciplinaridad}

El apoyo educativo interdisciplinario con población vulnerable, en este caso, con las personas menores institucionalizadas en los albergues del PANI, constituye un área de enorme crecimiento profesional que integra un espacio de atención que, de manera desafortunada, se vislumbra escasamente abordado, ya que no han podido determinarse estudios a nivel nacional o internacional orientados hacia el objetivo que esta propuesta plantea.

La propuesta pedagógica se orienta hacia una visión constructivista, obedece a lo indicado por Ortiz Granja (2015, p. 96):

El conocimiento es una construcción del ser humano: cada persona percibe la realidad, la organiza y le da sentido en forma de constructos ..., lo que contribuye a la edificación de un todo coherente que da sentido y unicidad a la realidad.

Como lo indica Ortiz Granja (2015), esta teoría procura la construcción y reconstrucción del conocimiento, de manera acorde con las realidades específicas de la población aprendiente, que reconstruye desde su realidad particular. Por tanto, la propuesta pedagógica no responde a un currículo determinado, sino a múltiples factores administrativos o educativos, como la alta movilidad de la población hacia otras alternativas de protección, ingreso a organizaciones no gubernamentales (ONG), reubicación familiar; así como los diferentes niveles de escolaridad, discapacidades, condiciones emocionales y de aprendizaje presentes, entre otros. 
http://doi.org/10.15359/ree.24-3.8

En este sentido, es cada profesional en educación quien, desde su disciplina, con orientación de un diagnóstico pedagógico, establece las necesidades y prioridades de trabajo con la población albergada; esto, en estrecha comunicación con las coordinadoras académicas de cada área, quienes le dan seguimiento al componente educativo propuesto por cada profesional.

Sin embargo, para plasmar claridad en la mediación pedagógica, la propuesta educativa responde a una serie de principios, uno de los más importantes es la interdisciplinariedad, entendida como: la cualidad del trabajo realizado a partir de la puesta en práctica de varias disciplinas; por ende, este concepto asume la existencia de un grupo de áreas del conocimiento relacionadas entre sí o de un conjunto de ciencias que logran vincularse para llevar a cabo acciones específicas. Cabe destacar que la interdisciplinariedad evita el desarrollo o ejecución de trabajos fragmentados por áreas del saber; por el contrario, ésta misma se conceptualiza como un proceso dinámico cuyo propósito principal es el desenvolvimiento científico y técnico de diversas ramas disciplinarias, que funcionan de forma integrada, para atender una necesidad o situación de manera acertada y efectiva.

[La interdisciplinariedad significa] un cambio de actitud frente a los problemas del conocimiento [y sustituye] ... la concepción fraccionada por una unitaria del ser humano y de las realidades que vive.

En esta dirección, si se desea superar la visión atomizada del conocimiento, se requiere repensar el ejercicio docente desde el diseño e implantación de estrategias que respondan a las necesidades del estudiantado y que propendan hacia el desarrollo de un pensamiento interdisciplinar. (Pirela-Morillo y Pico-Sánchez, 2007, p. 6)

Es fundamental para el equipo de profesionales del proyecto comprender que el trabajo interdisciplinario es necesario para la atención de la población menor de edad y el éxito en la atención educativa brindada, pues la realidad se concibe como una sola y no como un espacio fragmentado, y el aporte de cada disciplina enriquece el proceso de aprendizaje de cada menor de edad y le permite una visión más integral de su propio contexto y vivencias.

Por su parte, el hacer profesional le da un mayor sentido a su accionar pedagógico y lo aborda en función de las necesidades e intereses de la población atendida.

En esta línea, Tamayo y Tamayo (2011) divide la interdisciplinariedad de la siguiente manera:

Auxiliar: Se presenta cuando una disciplina recurre, permanente u ocasionalmente, a los métodos de otra u otras para el logro de su propio desarrollo.

Instrumental: Se presenta a partir de ciertos instrumentos metodológicos aplicables a diversas disciplinas, y que llegan a constituirse en objeto de estudio independiente. 
http://doi.org/10.15359/ree.24-3.8

http://www.una.ac.cr/educare

educare@una.cr

Estructural: Se presenta a partir del estudio de estructuras comunes que permiten estudiar conexiones en hechos distantes, catalogados en disciplinas distintas.

Conceptual: Cuando a partir de un concepto de carácter genérico, independiente de una disciplina específica, se aclaran fenómenos presentados en la realidad y que pueden ser estudiados por diversas disciplinas.

Operativa: Cuando el fenómeno de estudio debe ser analizado por especialistas de otras disciplinas y métodos en que han surgido, es decir, que se amplía el margen de fuentes de información.

Metodológica: Se presenta cuando el punto de partida o convergencia entre varias disciplinas es el método de trabajo, el cual facilita la interpretación de la realidad. La investigación interdisciplinaria a partir del método unifica criterios y reúne lo que en las disciplinas aparece disperso.

Limítrofe: Se presenta cuando los métodos y contenidos de dos o más disciplinas tratan un mismo tipo de fenómenos, considerados desde puntos de vista propios de cada disciplina y que presentan margen de coordinación, de posible transferencia de leyes, principios o estructuras de una a otra disciplina.

Teórica: Cuando una disciplina considera que los principios, leyes, axiomas y teorías han alcanzado niveles científicos más elevados que otros y, por tal razón, tratan de configurarse según los modelos de esa disciplina.

Compuesta: Se conforma para la búsqueda de solución de problemas de alta complejidad (ecológicos, demográficos, seguridad social, etc.) en los cuales deben intervenir diversas disciplinas, a fin de proyectar las alternativas de solución para el problema planteado. (pp. 19-20)

Dentro de la clasificación propuesta por el autor antes mencionado y con base en la realidad del proyecto, se utiliza la modalidad estructural, pues al ser el albergue un espacio con características muy definidas y con una población con particularidades similares, las problemáticas que se encuentran pueden ser analizadas por las diversas personas profesionales desde sus experiencias disciplinares. Asimismo, la modalidad metodológica les ha permitido identificar necesidades socioemocionales de la población desde diversas disciplinas, tales como autocontrol, motivación hacia el estudio, proyecto de vida, respeto hacia los demás, las cuales se han logrado abordar acordando un plan de acción que les permita trabajar sobre la necesidad de una forma conjunta. Además, el equipo de profesionales ha logrado trabajar interdisciplinariamente temas académicos al realizar procesos de forma conjunta in situ, o bien, 
http://doi.org/10.15359/ree.24-3.8

al lograr acuerdos que les permitan trabajar, desde la mediación pedagógica de su área, un mismo contenido, como el profundizar en el desarrollo motor de menores preescolares desde la educación física y la educación preescolar, o el desarrollo de contenidos matemáticos desde la música y la matemática.

Desde esta perspectiva, al aplicar la interdisciplinariedad se precisa de la visualización y el análisis de los aspectos e implicaciones que las diferentes disciplinas puedan aportar; esto, con el propósito de alcanzar una fusión conceptual y metodológica estable y compatible. En esta línea, a partir del trabajo interdisciplinario, Tamayo y Tamayo (2011) plantean que se deben establecer los siguientes indicadores:

a) el alcance de los objetivos planteados

b) la cantidad y calidad del trabajo realizado

c) el proceso y los productos desarrollados

d) la motivación hacia el trabajo en equipo

e) el sentido de pertenencia hacia su grupo

f) la eficacia y eficiencia del trabajo.

g) la valoración del trabajo mismo

Estos lineamientos requieren ser estudiados y analizados para establecer un proceso autoreflexivo acerca de su efectividad y de la integración de las disciplinas involucradas. En relación con los profesionales y las profesionales del proyecto, se considera que la motivación de trabajo con esta población específica marca mucho la diferencia en cuanto a intentar realizar acciones interdisciplinarias; adicionalmente, los equipos más estables, donde existe una menor rotación de profesionales, hace que las experiencias de trabajo conjunto sean acciones más elaboradas y más pertinentes.

Porotraparte,TamayoyTamayo (2011)indicanqueparahacerposiblelainterdisciplinariedad ciertas condiciones son necesarias en cada docente que se involucra:

a) dominar su disciplina (competencia: saber ser, conocer y hacer).

b) tener interés y comprensión del objetivo de la interdisciplinariedad.

c) realizar un trabajo a nivel conceptual y metodológico basado en el los objetivos establecidos.

d) mantener comunicación constante con el personal involucrado.

e) unificar esfuerzos entorno al diseño y realización del trabajo. 
http://doi.org/10.15359/ree.24-3.8

http://www.una.ac.cr/educare

educare@una.cr

Estas disposiciones determinan, en gran medida, la funcionalidad de la interdisciplinariedad con respecto a las diversas disciplinas. Finalmente, para que el trabajo interdisciplinario funcione de manera adecuada, se precisa que algunas barreras a nivel académico y cultural sean sobrepasadas, tales como el celo profesional tan comúnmente encontrado en las disciplinas educativas. En esta línea, Carvajal Escobar (2010) menciona que "se requiere cooperación, respeto mutuo, confianza y apertura mental para manifestar las complementariedades y lo que cada uno puede aportar a un proyecto interdisciplinario" (p. 162). De este modo, es importante destacar que la interdisciplinariedad requiere la voluntad de los participantes en donde se conjugan sus valores personales y una concepción curricular percibida desde una visión abierta y enfocada en el aprendizaje del estudiantado; por ende, desde la experiencia del proyecto es necesaria la capacitación en el modelo pedagógico utilizado y visualizar la necesidad de que exista una flexibilidad curricular con una visión transformable, que le permita a cada profesional, desde el marco y ética de su disciplina, tomar decisiones que orienten la mediación en pro de responder de una forma eficaz a las necesidades siempre cambiantes de la población atendida.

\section{Importancia de la interdisciplinariedad en contextos educativos}

En el análisis de este proyecto, destaca la importancia de referirse a la interdisciplinariedad como un elemento vital en la construcción de saberes, redireccionar los procesos de mediación hacia la construcción conjunta de conocimiento, estimular las comunidades de aprendizaje y el enriquecimiento profesional desde la propia perspectiva y el punto de vista del otro ser, presentar el aprendizaje con base en diversas visiones, donde su integración le da relevancia a la interdisciplinaridad y enriquece el bagaje integral de quien aprende.

Por ello, es fundamental entender la interdisciplinariedad como la estrategia óptima para que cada una de las disciplinas que intervienen en la formación del estudiantado aporte sus propios esquemas conceptuales, su manera de definir y abordar los problemas y sus propios métodos de investigación (López, 2012).

Por otra parte, para alcanzar una mediación efectiva es fundamental considerar lo indicado por Parra (2010, p. 122):

[Esta] permite expresar los dos planos tanto el interno como el externo y tiene su base en el lenguaje como recurso fundamental que se convierte a su vez en un medio de autorregulación y la forma de responder el estudiante frente a las diferentes situaciones de la cultura pedagógica y valores de los involucrados.

Por lo tanto, en los albergues, las múltiples destrezas y habilidades profesionales se conjugan para que desde su constructo disciplinar aporten soluciones a los diversos problemas,

8 Roxana Rodríguez-Araya, Priscilla Carranza-Marchena, Raquel Bulgarelli-Bolaños, Silvia Ulate-Oviedo y Maureen Camacho-Oviedo

Los artículos de la Revista Electrónica Educare del Centro de Investigación y Docencia en Educación de la Universidad Nacional, Costa Rica, se comparten bajo términos de la Licencia Creative Commons: Reconocimiento, № Comercial, Sin Obra Derivada 3.0 Costa Rica. Las autorizaciones adicionales a las aquí delimitadas se pueden obtener en el correo: educare@una.cr 
http://doi.org/10.15359/ree.24-3.8

conflictos o necesidades existentes, potenciando aprendizajes integrados y enfatizando así el abordaje holístico de la educación, a través de una mediación pedagógica adaptada a la realidad individual y contextual de las PME.

Por otra parte, un aspecto relevante que no se puede obviar es que el enfoque interdisciplinario no es habitual en la práctica pedagógica, muchos factores confluyen para que esté ausente; entre ellos destaca el hecho de que la mayoría de organizaciones posee una estructura disciplinar muy definitiva y marcada, cuya puesta en práctica implica horarios y espacios que, en la mayoría de ocasiones, ya están establecidos y son rígidos, lo cual dificulta que las diversas áreas confluyan naturalmente. No obstante, esto no debe invisibilizar el enriquecimiento que la interdisciplinariedad le otorga a la práctica pedagógica.

En la interdisciplinaridad deben promoverse todo tipo de ajustes y modificaciones que procuran la apertura de espacios de encuentro, donde se potencie la posibilidad de desarrollar formatos curriculares diversos, tomando en cuenta, por supuesto, que las dificultades organizacionales no son las únicas que impiden la práctica natural de la interdisciplinariedad, sino que el mayor reto a superar es el temor de salir de la propia zona de confort que promueve la formación disciplinar y trabajar en la aplicación de la perspectiva personal, que otorga la práctica en la propia área del saber, con la iniciativa de establecer diálogos, intercambios y acuerdos con colegas de otras disciplinas.

En este aspecto, el abordaje interdisciplinario del proyecto resulta vital al brindar espacios no estructurados de intercambio de conocimientos, más flexibles e integrados, contar con la posibilidad de ajustar tiempos, abordajes y objetivos, según las necesidades de las PME, y promover, no solo un aprendizaje integral y significativo, sino, además, un abordaje tendiente a "dar al educando una formación de calidad, íntegra y holística que solo se consigue mediante la educación interdisciplinaria" (López, 2012, p. 368).

\section{Ruta metodológica}

El tipo de estudio que se presenta es cualitativo y de enfoque descriptivo, debido a que "proporciona profundidad a los datos, dispersión, riqueza interpretativa, contextualización del ambiente o entorno, detalles y experiencias únicas. Asimismo, aporta un punto de vista 'fresco, natural y holístico' de los fenómenos, así como flexibilidad" (Hernández Sampieri et al., 2014, p. 16). Se presenta como enfoque descriptivo al permitir lo siguiente:

La posibilidad de generalizar los resultados más ampliamente, otorga control sobre los fenómenos, así como un punto de vista basado en conteos y magnitudes. También, brinda una gran posibilidad de repetición y se centra en puntos específicos de tales fenómenos, además de que facilita la comparación entre estudios similares. (Hernández Sampieri et al., 2014, p.15) 
http://doi.org/10.15359/ree.24-3.8

http://www.una.ac.cr/educare

educare@una.cr

Desde esta propuesta, el equipo de profesionales desarrolla una estrategia de trabajo interdisciplinario mediante la ejecución pedagógica establecida por el proyecto. Esta ejecución se refiere a la propuesta que desarrollará cada profesional y debe considerar los siguientes elementos: la formación profesional, las necesidades y habilidades de la población menor de edad y la realidad del albergue (UNA, Costa Rica, 2017).

\section{Participantes}

En relación con el conjunto de participantes del proyecto, es importante aclarar que está constituido por el equipo de profesionales contratado para dar servicios pedagógicos en los albergues y aldeas del PANI durante los años 2016, 2017, y 2018.

Se deben comprender los sujetos participantes en dos dimensiones. La primera relacionada con participantes que proporcionaron información en las encuestas realizadas anualmente y, en segundo lugar, las participaciones de profesionales en las reuniones bimensuales del equipo de cada albergue; para esto se realizó el llenado de una minuta por reunión definida por la coordinación del proyecto, con el fin de comprender el trabajo realizado y los acuerdos tomados conjuntamente.

En el caso de las encuestas, se contó con la participación de profesionales, que se contrataron, de las diferentes áreas pedagógicas que ofrece el proyecto. En la primera encuesta del 2016, se contó con el aporte de 66 profesionales; para la segunda, en el 2017, participó un total de 95 profesionales, y en la tercera encuesta, en el 2018, se logró la participación de 123 profesionales. En cuanto a las reuniones del equipo interdisciplinario se contó con 63 participaciones en el 2016 y con 322 en el 2017.

\section{Técnicas e instrumentos}

Es interés del equipo coordinador del proyecto valorar la labor pedagógica desarrollada en los albergues, específicamente desde el trabajo interdisciplinario, para ello, se realizan dos acciones que permiten recabar los insumos necesarios. Una primera acción corresponde a la técnica de la encuesta aplicada en tres momentos, definidos en el año 2016, 2017 y 2018, y una segunda acción de sistematización de las minutas de reunión de los equipos interdisciplinarios de los albergues durante el 2016 y 2017. Se escoge esta técnica debido a que el proyecto abarca todo el territorio nacional; por tanto, toda la logística administrativa se realiza de forma virtual.

[La encuesta] se basa en el interrogatorio de los individuos, a quienes se les plantea una variedad de preguntas con respecto a su comportamiento, intenciones, actitudes, conocimiento, motivaciones, así como características demográficas y de su estilo de vida. (Malhotra, 2008, p. 183) 
http://doi.org/10.15359/ree.24-3.8

Además, cabe mencionar que estas encuestas fueron aplicadas de dos formas: de manera digital, por medio de los formularios de Google, se aplicaron dos de ellas en el 2016 y 2017, y la última en el 2018, de manera presencial.

Las preguntas eran de tipo abierto, para realizar posteriormente un análisis del contenido de las respuestas desde el enfoque cualitativo, y orientadas a conocer las razones por las cuales se lograban o no realizar las acciones de trabajo interdisciplinario. A continuación, se detallan cada uno de los momentos de aplicación de la encuesta utilizada, cuya importancia es la recolección de información valiosa para la mejora continua del proyecto.

\section{Encuesta de diciembre de 2016}

Esta encuesta, realizada en el 2016, constituye el primer momento y se elabora con el objetivo de valorar la percepción del trabajo realizado y los logros alcanzados por el trabajo en el proyecto de octubre 2016 a diciembre 2016. La encuesta es un instrumento que se elabora en línea, mediante el sistema de formularios de Google, en su construcción, envío, resolución y sistematización.

Este documento se envía en línea a todo el equipo de profesionales, mediante sus correos electrónicos y se da seguimiento a su llenado durante un lapso de un mes, aproximadamente. Valora aspectos relacionados con datos básicos (se refiere a la disciplina), el trabajo con las personas menores, administración (en función de la comunicación con diferentes figuras del proyecto), satisfacción laboral y un último espacio de observaciones.

Esta encuesta consta de 36 preguntas, de las cuales se recoge la información arrojada por la pregunta número 9, la cual se basa en el trabajo interdisciplinario, específicamente, al consultar: ¿Se logró realizar trabajo interdisciplinario con los diferentes profesionales que laboran en el albergue? Justifique su respuesta. La información se sistematiza y se desarrolla en el apartado de análisis correspondiente.

\section{Encuesta de enero de 2017}

Esta segunda encuesta abarca el segundo momento y también se plantea con el propósito de valorar la percepción del trabajo realizado y los logros alcanzados por el proyecto, pero en un periodo mayor de tiempo, pues abarca lo trabajado desde enero 2017 a diciembre 2017. Este instrumento se elabora en línea, mediante el sistema de formularios de Google, al igual que la encuesta 2016, se construye, envía, soluciona y sistematiza mediante esta herramienta tecnológica.

El documento se envía mediante correo electrónico a todo el equipo de profesionales que trabajaron durante el 2017 y se le da seguimiento a su llenado durante un lapso de tiempo establecido de quince días. La encuesta aborda temáticas a valorar similares a las planteadas en la encuesta de diciembre 2016. Esta encuesta estaba constituida por 21 preguntas, de las cuales, 
http://doi.org/10.15359/ree.24-3.8

http://www.una.ac.cr/educare

educare@una.cr

para el análisis del trabajo interdisciplinario, se recoge la información de la pregunta número 5 , que consulta sobre: ¿Se logró realizar trabajo interdisciplinario con los diferentes profesionales que laboran en el albergue? Justifique su respuesta La información de esta encuesta se unifica con la información sistematizada del año 2016 y se considera en el apartado de análisis correspondiente.

\section{Encuesta de enero y febrero de 2018}

En este tercer momento, la encuesta fue distribuida en físico a profesionales que asistieron a los procesos de inducción desarrollados por las coordinadoras del proyecto, durante enero y febrero 2018. Consta de 8 preguntas que, en general, consultaban sobre la percepción respecto a lo acontecido mientras se ejecutaba el proyecto en temáticas como relaciones interpersonales de profesionales con personal colaborador del proyecto (PME, encargadas de cuido, fiscalizadoras y coordinación del proyecto), herramientas pedagógicas brindadas en la formación docente, aporte de los materiales en la función pedagógica y experiencias pedagógicas significativas o interdisciplinarias.

En particular se aborda el análisis de la pregunta número 7: Coméntenos sobre una experiencia pedagógica interdisciplinaria que haya vivenciado en su labor con las PME. Parte de esta dinámica de inducciones se realizó en las instalaciones del Centro de Investigación y Docencia en Educación (CIDE) de la Universidad Nacional (UNA) y otras en las diversas regiones donde se desarrolla el proyecto y que están a mayor distancia de la UNA.

Estas reuniones surgen por el interés de brindar espacios de contacto más directo con los equipos profesionales y conocer de primera instancia sus percepciones, necesidades y recomendaciones en cuanto a la ejecución del proyecto. En las reuniones organizadas se contó con la participación de la mayoría de profesionales de las distintas regiones atendidas y que laboraron durante el 2018. Incluso se realizó una sesión adicional de inducción para que asistieran las personas profesionales que por alguna razón no pudieron asistir a la reunión regional correspondiente. Por lo anterior, se aprovechó este espacio de socialización para desarrollar la encuesta de forma general. La información contemplada en el análisis sobre el trabajo interdisciplinario y las sesiones de inducción permitieron valorar los alcances del proyecto, aclarar consultas, fomentar el trabajo en equipo y dar directrices con respecto al accionar del proyecto durante el 2018.

\section{Minutas de reunión del equipo de albergues 2016-2017}

La segunda acción realizada para el estudio y que constituye una fuente importante de información del trabajo interdisciplinario que se utilizó fueron los insumos sistematizados por el mismo grupo de profesionales en las minutas de reunión de los equipos por albergue desde octubre de 2016 hasta diciembre de 2017. Estas reuniones son organizadas a lo interno de cada equipo de albergue y se puntualizan los temas tratados en una minuta de reunión, la cual 
posteriormente es colocada en la carpeta de información digital del proyecto. Esta labor es parte de las funciones de la persona profesional en orientación de cada albergue, quien sistematiza en la minuta los asuntos de reunión y los hace visible para las coordinadoras del proyecto, al colocar la información en la carpeta digital que se encuentra en la página Web del proyecto.

Estas reuniones son autogestionadas por cada equipo de albergue y en algunas de ellas pueden participar las fiscalizadoras de los albergues establecidas por el PANI. Parte de la información de esas minutas corresponde al trabajo interdisciplinario y a las experiencias de trabajo integrado que como equipo de albergues han desarrollado. Esta información es la que se considera como otro insumo para el análisis planteado. En total se contó con la información de 108 minutas de reunión de equipo de albergue en las diferentes regiones; 45 de ellas recopiladas entre octubre y diciembre del 2016, y las restantes 63 recabadas entre febrero y diciembre del año 2017.

Se debe comprender que una minuta es un recurso escrito que facilita sistematizar los asuntos de relevancia tratados en una reunión, proporciona una descripción de la reunión y sus principales planteamientos o conclusiones, por esto favorece la organización de las temáticas de interés y permite fomentar el trabajo interdisciplinario, así como lograr metas comunes, integrar grupos de trabajo más eficaces, pasar de un accionar individual a una actuación de equipo, crear experiencias individuales, pero también colectivas. Asimismo, estas experiencias e insumos conllevan a responder, de forma más eficiente, a las necesidades detectadas en el desarrollo del apoyo pedagógico, ampliar talentos y enriquecer la formación y actuar de cada profesional del proyecto. La información recolectada en las minutas fue sistematizada en una matriz, en la cual se categorizan las acciones planteadas por los equipos de los albergues para, posteriormente, realizar un análisis del contenido de las respuestas.

\section{Resultados y discusión}

A partir del desarrollo de este proyecto pedagógico y de su propuesta de trabajo interdisciplinario, es importante reflexionar sobre los resultados obtenidos.

\section{Complejidad del proceso y la evaluación permanente}

El proyecto pedagógico propuesto es complejo, debido a varias condiciones relacionadas con su ejecución, como los múltiples niveles de comunicación requeridos entre instituciones, unidades académicas y profesionales; la cobertura amplia al hacer un abordaje a nivel nacional; una población infantil y adolescente diversa por atender; una propuesta curricular flexible y significativa en el desarrollo integral de los menores de edad, y un equipo de profesionales de múltiples disciplinas que requieren trabajar en un mismo espacio de forma integrada, pero no necesariamente coincidiendo en horario. Por tanto, surge la necesidad de realizar una evaluación 
http://doi.org/10.15359/ree.24-3.8

http://www.una.ac.cr/educare

educare@una.cr

constante del proceso. Dada la complejidad, es fundamental la evaluación constante que brinde datos para conocer los impactos alcanzados y guiar la toma de decisiones, y el análisis de las mejoras requeridas.

\section{Propuesta de trabajo interdisciplinario}

Al considerar la interdisciplinariedad, es valioso comprender los resultados de la información recabada al respecto. En el periodo de octubre a diciembre 2016, se recopila información relacionada con la temática de este artículo y se visualiza que la mayoría de los sujetos encuestados (73\%) afirma que fue posible realizar actividades interdisciplinarias, mientras que un $27 \%$ responde negativamente a esta misma interrogante.

Al analizar las razones de este dato sobre el trabajo interdisciplinario, se identifican cuáles se vinculan con la ejecución de la propuesta pedagógica interdisciplinaria y cuáles afectan esta ejecución. Entre los motivos por los cuales los equipos de profesionales afirman que fue posible el trabajo interdisciplinario se establecen los siguientes:

- Buena comunicación entre el equipo base en los albergues.

- Coincidencia en horarios.

- Disposición y colaboración del equipo.

- Asistencia de los profesionales a las reuniones pactadas.

- Realización de talleres y actividades de cierre del año.

- Necesidades de la población menor de edad.

Contrariamente, quienes indican que no lograron realizar el trabajo en esta modalidad, resaltan motivos como los siguientes:

- Poco encuentro de horarios entre profesionales.

- Preocupación por cumplir los objetivos de cada área.

- Baja planificación grupal.

- Poca comunicación entre compañeros y compañeras.

- Población infantil o adolescente reducida y transitoria.

Como puede verse, quienes realizan actividades interdisciplinarias resaltan, como fundamental, la existencia de comunicación, disposición, colaboración y compromiso entre el equipo de profesionales, lo cual es consistente con lo indicado por Tamayo y Tamayo (2011) 
http://doi.org/10.15359/ree.24-3.8

cuando indica que se requieren condiciones tales como tener interés y comprensión del objetivo de la interdisciplinariedad, mantener comunicación constante con el personal involucrado y unificar esfuerzos en torno al diseño y realización del trabajo.

Por lo contrario, la minoría de la población encuestada encuentra dificultades en el trabajo interdisciplinario lo aduce a razones como la preocupación de no cumplir con sus objetivos disciplinares, lo cual indica que esas personas no tienen una claridad de los fines de la interdisciplinariedad y sus alcances; priorizan sus disciplinas por sobre el trabajo interdisciplinario, pues losobjetivos de cadaárea pueden lograrse desde la colaboraciónyla coordinación, alcanzando un impacto mayor de la integración de saberes. Adicionalmente, en la segunda evaluación realizada a profesionales a finales del año 2017, se amplía el logro del trabajo interdisciplinario, pues 95 participantes que completaron la encuesta manifestaron que la experiencia de trabajo interdisciplinario había mejorado en relación con el periodo anterior. A este respecto, el $76 \%$ del equipo de profesionales afirmó que les fue posible realizar este tipo de acciones, mientras que un 24 \% respondió negativamente a la realización de estrategias interdisciplinarias.

El equipo de profesionales asevera, en esta segunda valoración, que fue posible el trabajo interdisciplinario por estas razones:

- Coincidencia en horarios.

- Asistencia a reuniones.

- Disposición y colaboración del equipo.

- Buena comunicación

- Reconocimiento de las aptitudes.

- Experiencia de cada miembro del equipo.

- Realización y participación en actividades recreativas, reuniones y talleres.

Por otro lado, entre los motivos para la no realización del trabajo en esta modalidad se resaltan como más frecuentes los siguientes:

- Poco encuentro en horarios.

- Poca comunicación entre profesionales.

- Poca accesibilidad de los compañeros y compañeras.

- Baja planificación grupal.

- Preocupación por cumplir los objetivos de cada área. 
http://doi.org/10.15359/ree.24-3.8

http://www.una.ac.cr/educare

educare@una.cr

Estos motivos encontrados, tanto de quienes realizan trabajo interdisciplinario, como de quienes no lo hacen, son consistentes con los encontrados en el año anterior (2016), lo cual indica que continúa existiendo la necesidad de trabajar con los equipos la importancia de la interdisciplinaridad y profundizar en los beneficios que estas actividades sugieren para la población apoyada. Es decir, se recalca que para alcanzar un trabajo interdisciplinario se requiere un equipo de profesionales consciente de la importancia de la comunicación entre docentes que dan servicio en cada albergue, además de estar anuentes a vivenciar experiencias conjuntas que les den experticia en estos procesos. Esto es congruente con lo que indica Carvajal Escobar (2010) sobre la importancia de la cooperación y apertura para trabajar interdisciplinariamente.

Por su parte, las razones que dificultan la ejecución del trabajo interdisciplinario refuerzan la importancia de lograr mayor coincidencia horaria entre el equipo de profesionales para ampliar las posibilidades de coordinación en las áreas de especialidad, que a sus vez, permitan el desarrollo de cada área disciplinaria y la integración de los conocimientos para un aprendizaje más significativo por parte de los menores de edad.

\section{Valoración del trabajo interdisciplinario}

En las inducciones realizadas en el año 2018, se retomó el tema del trabajo interdisciplinario como uno de los elementos de evaluación del año anterior, pues, al ser la interdisciplinariedad un principio del proyecto, se requiere evaluar desde la praxis el alcance de esta propuesta. De los 123 profesionales que participaron del proceso, un $96 \%$ resalta la importancia del trabajo interdisciplinario. Realizan algunas opiniones que le dan sustento a la estrategia; por ejemplo, un docente acota el apoyar a las personas menores de edad de manera interdisciplinaria ha sido una experiencia maravillosa, ya que todos estamos en sintonía con los procesos de las PME y de esta forma los mismos muestran mayores avances; verlos que muchos suben rendimiento escolar, modifica conductas, muestran mayor respeto (Profesional 06).

Esto permite identificar que el beneficio en el proceso es integral para la persona menor de edad, pues le permite, no solo avances de carácter académico, sino también en el área social. A su vez, estas experiencias interdisciplinarias fortalecen el accionar profesional, pues se logra atender más eficientemente el proceso pedagógico de menores de edad y también desarrollan herramientas de trabajo esenciales, que les permitirán fortalecer sus aptitudes laborales dentro del proyecto como tal, así como a futuro en otros campos de acción docente.

En relación con los alcances, los mismos grupos profesionales plantean mejoras referentes a:

a) la convivencia de las PME: se trabajó, en varias ocasiones, de manera interdisciplinaria y esto ha sido de gran ayuda para que las PME convivan e interactúen de manera saludable a la vez que aprenden mucho. (Profesional 03) 
http://doi.org/10.15359/ree.24-3.8

b) el trabajo de los profesionales: ... es más sencillo en la distribución de tareas o funciones. (Profesional 19)

c) interacciones personales de los docentes: creo que ... se logró crear un trabajo interdisciplinario, ya que las compañeras son muy anuentes a colaborar y a trabajar en equipo. (Profesional 28)

Estos tres beneficios indicados posibilitan comprender que, si se logra establecery apoyar las estrategias del trabajo interdisciplinario, se pueden alcanzar aprendizajes valiosos e importantes para las personas que colaboran y para las PME que reciben el servicio educativo. Surge como un pilar fundamental el trabajo en equipo para la planificación de las acciones docentes.

\section{Experiencias de trabajo interdisciplinario}

Dada la relevancia del principio de interdisciplinariedad en la labor pedagógica del proyecto, es importante destacar las principales acciones pedagógicas de esta índole desarrolladas por los equipos de albergue. Así que, al consultarle al equipo de profesionales sobre cuáles experiencias concretas de trabajo interdisciplinario desarrollaron, se evidencia una gran gama, las cuales incluyen:

a) Talleres. Por ejemplo: Los talleres interdisciplinarios dejan muchos aprendizajes en ambas partes, cuando se trabaja en conjunto, las PME se unen también ... (Profesional 19)

b) Actividades conmemorativas o fechas importantes (día del niño, actividades navideñas, entre otros). Por ejemplo: Una de las tantas experiencias que tuvimos fue la del día del niño, ..., nos distribuimos en sectores y la convivencia fue asombrosa, se realizó un trabajo generalizado, con los compañeros del proyecto. (Profesional 11)

c) Análisis de situaciones difíciles. Por ejemplo: Un niño ha sido muy difícil ..). Para evitar que se distanciara de mí, indagué con los demás profesionales cuáles eran sus intereses, así que basé en eso cada sesión y ahora nos llevamos muy bien y él siempre tiene interés y deseo de las sesiones. (Profesional 30)

d) Apoyo en la mediación. Por ejemplo; En orientación y con docentes de física se trabajan juegos dinámicos para el manejo de emociones como la frustración, y, en la parte vocacional, se trabaja en cine foros las formas de relacionarse positivamente. (Profesional 56)

e) Proyectos para el albergue. Por ejemplo: se planteó la oportunidad de realizar una huerta con los jóvenes del albergue, de manera que se realizó una serie de acciones para conseguir los materiales necesarios y se trabajó con los muchachos durante todo el proceso. (Profesional 97) 
http://doi.org/10.15359/ree.24-3.8

http://www.una.ac.cr/educare

educare@una.cr

f) Crecimiento profesional. Por ejemplo: Elapoyaralos menores demanera interdisciplinaria ha sido una experiencia maravillosa, ya que todos estamos en sintonía con los procesos de los menores y de esta forma los mismos muestran mayores avances; verlos que muchos suben rendimiento escolar, modificar conducta. (Profesional 06)

Todas estas acciones permiten comprender que los beneficios de la interdisciplinaridad pueden ser muchos y variados, si se logra salir del esquema tradicional y se apuesta por trabajos más holísticos que mejoren la experiencia educativa. El tipo de acciones interdisciplinarias son muchas y variadas, e implican un aprendizaje de profesionales sobre nuevas formas de gestionar los procesos de mediación, lo que deriva en un beneficio directo para la población infantil y adolescente. Se reflejan experiencias vinculadas al área académica, pero también relacionadas con la mediación pedagógica, las metodologías creativas y la valoración de la persona en su integralidad dentro del proceso, y no solo desde el aspecto académico.

\section{Espacios de reunión}

Un aspecto relevante de un proceso pedagógico es el generar espacios de reunión para el intercambio de ideas, la escucha activa de miembros del equipo, la atención de situaciones, la reflexión sobre las vivencias y la toma de decisiones. Al respecto, el proyecto siempre hizo hincapié en la importancia de identificarse como equipo y generar momentos de reunión para la construcción, como profesionales, de cada albergue y la toma de decisiones.

En la revisión de las minutas de reuniones interdisciplinarias, en el período de trabajo de dos meses del año 2016, los equipos de profesionales registraron 45 reuniones en total con representación de todas las Direcciones Regionales donde se ubicó el proyecto; esto, con un promedio de dos reuniones por albergue. Por otra parte, en el año 2017, en un periodo de trabajo de 11 meses, se registraron 63 minutas de reunión; de igual manera, con un promedio de dos reuniones por albergue con representación de casi la totalidad de las Direcciones Regionales con las que se inició en el 2016. Se aclara que para el 2017, se incorporó la participación del equipo de la Región Huetar Norte.

Las reuniones se convirtieron en espacios para abordar diferentes temáticas de interés del equipo de profesionales. Basándose en estas reuniones interdisciplinarias, según el orden de frecuencia, en la Tabla 1 se sistematizan los principales temas tratados, los cuales fueron reiterativos en el 2016 y en el 2017, interpretándose que estas temáticas fueron importantes para los grupos profesionales, y les generaron experticia y acciones creativas y reflexivas. Las principales temáticas abordadas en ambos periodos se resumen en la Tabla 1: 
http://doi.org/10.15359/ree.24-3.8

Tabla 1: Bitácoras de reuniones interdisciplinarias realizadas por los equipos

Temática

Disposiciones de logística y administrativas requeridas

Estrategias pedagógicas interdisciplinarias para atender problemas emocionales y de conducta.

Organización de talleres, proyectos y actividades recreativas de forma interdisciplinaria en temas diversos.

Líneas pedagógicas en común para trabajar con el personal del PANI o en relación con este.

Acciones para reforzar el trabajo en equipo.

Análisis de casos de las PME a nivel pedagógico.

Estrategias pedagógicas interdisciplinarias para atender problemas académicos.

Líneas pedagógicas en común para trabajar casos de personas con discapacidad.

Estrategias pedagógicas interdisciplinarias para promover la formulación de proyectos de vida en las PME.
Año 2016 Año 2017

$22 \quad 45$

$21 \quad 12$

$20 \quad 22$

$20 \quad 13$

$14 \quad 12$

$10 \quad 32$

$5 \quad 15$

10

$1 \quad 6$

Nota: Elaboración propia.

Con base en la Tabla 1, es evidente que los equipos de profesionales señalan relevante la logística administrativa como un tema importante de abordar en las reuniones; para esto han establecido estrategias como la creación de grupos de mensajería en la plataforma de WhatsApp, para mantenerse en comunicación de forma constante. Pues, para el logro de la labor interdisciplinaria, los equipos de profesionales señalaron la comunicación como un elemento relevante para lograr la organización de las acciones que requieren del aporte del grupo y destacan el uso de otros medios de comunicación eficientes cuando no lograban la coincidencia horaria.

Porotraparte,elanálisis decasosseincrementóexponencialmentedebidoalaparticularidad de la población que se atiende y a sus necesidades, que se identifican constantemente, lo que indica un interés en promover una atención ajustada a la individualidad de la población. Esto generó un enriquecimiento profesional, pues se aprende a aportar desde el área de formación y a escuchar y aprender de otras profesiones, con el propósito fundamental de atender a la PME desde su situación personal y sus necesidades en diferentes dimensiones. Además, este análisis de casos se relaciona con otro punto relevante, que es la formulación de estrategias pedagógicas interdisciplinarias basadas en la atención de dificultades académicas de las PME. Esto, debido al rezago escolar, discapacidades y a otros factores que inciden directamente en el avance académico de la población; sin embargo, a raíz del apoyo de profesionales se denotan mejoras en las calificaciones y en la culminación del año escolar de las PME. 
http://doi.org/10.15359/ree.24-3.8

http://www.una.ac.cr/educare

educare@una.cr

Por otra parte, se denota una disminución considerable con respecto a las estrategias pedagógicas vinculadas con la atención de problemas emocionales y de conducta, lo cual se considera muy positivo; algunas causas probables relacionadas con esta mención se basan en el hecho de que el proyecto ha estado en funcionamiento por un periodo de tiempo más extenso, lo que genera una mejora más evidente en la convivencia y participación en los albergues. Esto refuerza el hecho de que el abordaje pedagógico debe ser integral y buscar la mejora del ser humano en sus diferentes dimensiones, y queda claro que los avances en un área de acción, con un trabajo interdisciplinario, va a conllevar a un enriquecimiento de otros ámbitos de la vida de la PME.

Se mantiene, de forma similar en las conversaciones de reunión de los equipos, la planeación de talleres, proyectos y actividades recreativas. Estas actividades se realizan en diversos momentos del año, pero se logra identificar que se desarrollaron mayoritariamente durante los periodos de vacaciones y efemérides, como el Día del Niño y la Niña y la Navidad. Si bien estas estrategias pueden estar vinculadas con períodos o fechas específicas, éstas son importantes para promover una convivencia armoniosa dentro de los albergues y aportar a una visión más amplia de aprendizaje; de esta manera, se analiza la forma de impulsar el incremento de estas estrategias desde la coordinación del proyecto, por lo que compartir las experiencias exitosas alcanzadas se identifica como una opción multiplicadora para incorporarlas con otros equipos de albergues.

En conclusión, las minutas de reuniones de equipo dilucidan el aumento en la mención de temáticas basadas en la coordinación de acciones administrativas, el análisis de PME que requieren una atención particular y más integral, el rendimiento académico y las estrategias pedagógicas interdisciplinarias que promueven un aprendizaje más holístico, como las principales acciones mencionadas.

\section{Informes integrados}

Adicionalmente, cabe mencionar que en el 2018, la coordinación del proyecto realizó un paso más en la dirección de la interdisciplinaridad, al impulsar la construcción de informes pedagógicos de las PME de forma conjunta. En los años 2016-2017, cada profesional de forma disciplinar elaboraba los informes de inicio, de avance, y de salida de cada PME, de manera que cada PME contaba con tantos informes como áreas disciplinarias hubiera recibido. Para el año 2018, se visualiza la necesidad de mejorar esta estrategia y cada profesional realiza solo un informe para cada PME, pero de manera integrada. Esto requirió de varios pasos previos, como la elaboración de una nueva plantilla de informe que lograra sistematizar la información pedagógica de cada menor de edad; el aval de los personeros del PANI para visualizar la opción de un informe más integrado y menos segmentado; la habilitación en la página web del proyecto pedagógico de un espacio en Google Drive que permitiera realizar las acciones requeridas para la 
formulación completa e integrada del informe; y la capacitación al equipo de profesionales para el uso de la plantilla en línea. Para lograr lo anterior, se requiere mucha coordinación de las personas encargadas del proyecto, como de los equipos de profesionales para generar un producto en conjunto, que logre evidenciar los avances y retos de la PME en las diferentes áreas de atención pedagógica. Ello recalca la importancia de valorar a la persona como un ser integral y que los servicios de apoyo educativos se constituyen en elementos potenciadores para cada PME.

\section{Discusión y conclusiones}

La interdisciplinariedad supone, en sí misma, un reto, pues la formación y la praxis cotidiana apuntan y fomentan acciones individuales; sin embargo, este proyecto ha dado un paso al plantear, desde sus principios y sus prácticas, acciones que la promuevan. Se partió desde la interdisciplinariedad como un principio en pro del fortalecimiento de las habilidades tanto académicas como emocionales de la población menor de edad atendida. Por ende, fue de vital importancia alejarse del modelo tradicional y acercarse a una realidad en la cual se conjugaron diversas áreas disciplinarias, cuyo fin común fue promover y potenciar el bienestar de las personas menores de edad con quienes se trabajó.

Asimismo, las dinámicas siempre cambiantes de los albergues, de la población que reside y de las encargadas de cuido directo hacen que se requiera una propuesta pedagógica abierta y flexible que logre comprender la cultura de cada alternativa de cuido. En este sentido, el trabajo interdisciplinario fue promovido y evaluado, de forma constante, por medio de la coordinación del proyecto; esto, con el objetivo de brindar seguimiento al equipo de profesionales base de cada albergue y así redireccionar las acciones y la toma de decisiones conjuntas.

Se plantea el abordaje interdisciplinario como mecanismo y recurso de apoyo adicional a la formación disciplinar. Asimismo, se potenciaron, por parte de los equipos base de los albergues, actividades distintas para promover y fortalecer el desarrollo de talentos y del disfrute de las PME. Por lo tanto, se requirió que los profesionales llegaran a acuerdos entre sí, en relación con las formas de abordar situaciones particulares de las $\mathrm{PME}$, en la mediación pedagógica, en la construcción de informes, en la formulación de talleres u otras iniciativas que mejoren la vida de los niños, niñas y adolescentes.

Con base en el análisis de datos realizado y en los resultados obtenidos, se destaca que la mayoría de profesionales visualizan positivamente la oportunidad de realizar acciones interdisciplinarias. Además, que este trabajo requiere de la voluntad de todas las personas participantes con respecto a su concepción, y visión curricular y profesional. Por tanto, se debe trabajar desde la capacitación y apertura hacia un modelo pedagógico diverso, en el cual la flexibilidad y transformación son aspectos claves en la orientación de una mediación pedagógica más abierta y enfocada en la persona menor de edad atendida, y en sus intereses y necesidades. 
http://doi.org/10.15359/ree.24-3.8

http://www.una.ac.cr/educare

educare@una.cr

Asimismo, cabe señalar que, mediante el trabajo interdisciplinario, es necesario realizar ajustes y modificar la forma de trabajo curricular, para así adaptar los espacios de encuentro hacia formas diversas de aprendizaje. De hecho, con base en este estudio, se identificaron características relevantes con respecto al trabajo interdisciplinario, entre las cuales están: la buena comunicación y compromiso entre el equipo docente, la flexibilidad curricular, la convivencia, las interacciones personales adecuadas, además del interés por apoyar los procesos de las PME como aspectos fundamentales para lograr realizar el trabajo interdisciplinario.

Por todo lo anterior, como parte del proceso de valoración de la propuesta educativa, se considera acertada la estrategia de interdisciplinariedad, pues la mayoría del grupo de profesionales participantes ha logrado asumir el compromiso de mirar más allá de su disciplina y mostrar disposición y colaboración en las acciones planteadas por el mismo equipo de trabajo, en pro de la mejora de la calidad de vida y experiencia educativa de niños, niñas y adolescentes que reciben el apoyo pedagógico.

La recomendación principal que arroja este estudio para la educación es mirar la interdisciplinariedad como una oportunidad de crecimiento pedagógico desde los múltiples saberes profesionales que se conjugan en una tarea específica. Se retoman las palabras de Carvajal Escobar (2010): "la interdisciplina, más que un término, debe de ser una estrategia pedagógica, que conceptualice los propósitos y la planificación del proceso" (p. 166). Por tanto, sólo trascendiendo la visión fragmentada del acto educativo se lograrán resultados más pertinentes en relación con las necesidades y realidades del estudiantado.

\section{Referencias}

Carvajal Escobar, Y. (2010). Interdisciplinariedad: Desafío para la educación superior y la investigación. Revista Luna Azul, 31, 156-169. http://www.scielo.org.co/pdf/luaz/n31/ $\underline{\text { n31a11.pdf }}$

Hernández Sampieri, R., Fernández Collado, C. y Baptista Lucio, P. (2014). Metodología de la investigación (6. ${ }^{\mathrm{a}}$ ed.). McGraw-Hill.

López, L. (2012). La importancia de la interdisciplinariedad en la construcción del conocimiento desde la filosofía de la educación. Sophia, Colección de Filosofía de la Educación, 13, 367377. http://www.redalyc.org/pdf/4418/441846102017.pdf

Malhotra, N. K. (2008). Investigación de mercados: Un enfoque aplicado (5. a ed.). Pearson Educación. https://www.academia.edu/15023888/Investigacion de Mercados - Naresh Malhotra

Ortiz Granja, D. (2015). El constructivismo como teoría y método de enseñanza. Sophia. Colección de filosofía de la educación, 19, 93-110. https://doi.org/10.17163/soph.n19.2015.04 
http://doi.org/10.15359/ree.24-3.8 http://www.una.ac.cr/educare educare@una.cr

Parra, K. (2010). El docente de aula y el uso de la mediación en los procesos de enseñanza y aprendizaje. Investigación y Postgrado, 25(1), 117-143. http://revistas.upel.edu.ve/index. php/revinpost/article/view/1357/531

Pirela-Morillo, J. y Pico-Sánchez, W. (2017). Lectura y pensamiento interdisciplinar en las clases de matemáticas y ciencias de la información. Revista Electrónica Educare, 21(3), 1-14. https:// doi.org/10.15359/ree.21-3.16

Tamayo y Tamayo, M. (2011). La interdisciplinariedad. ICESI.

Universidad Nacional, Costa Rica. (2017). Manual para la contratación de servicios de profesionales. Proyecto pedagógico para las personas menores de edad ubicadas en albergues institucionales del PANI. Manuscrito inédito. 